\title{
GENOMICS
}

\section{Mining for natural products}

Using phage display and a biotin-based selection system, researchers clone genes involved in natural product biosynthesis in bacteria.

The vast majority of microorganisms that inhabit the planet—several thousand species of bacteria per gram of soil alone-are thought to be unculturable in the laboratory. These constitute a great untapped resource for natural products, and recent work by researchers at Harvard Medical School, though still at the proof-of-concept stage, suggests one way that may bring us closer to mining this promising seam.

Polyketides and nonribosomal peptides are two major classes of natural products with important properties. Even unculturable species of bacteria are likely to have biosynthetic gene clusters corresponding to these classes of molecules, as these clusters have been found in organisms in every niche examined thus far. "These gene clusters share an assembly-line logic," says Jun Yin, now at the University of Chicago, and first author on the paper describing this work, "and the assembly line has carrier proteins that move things along." The

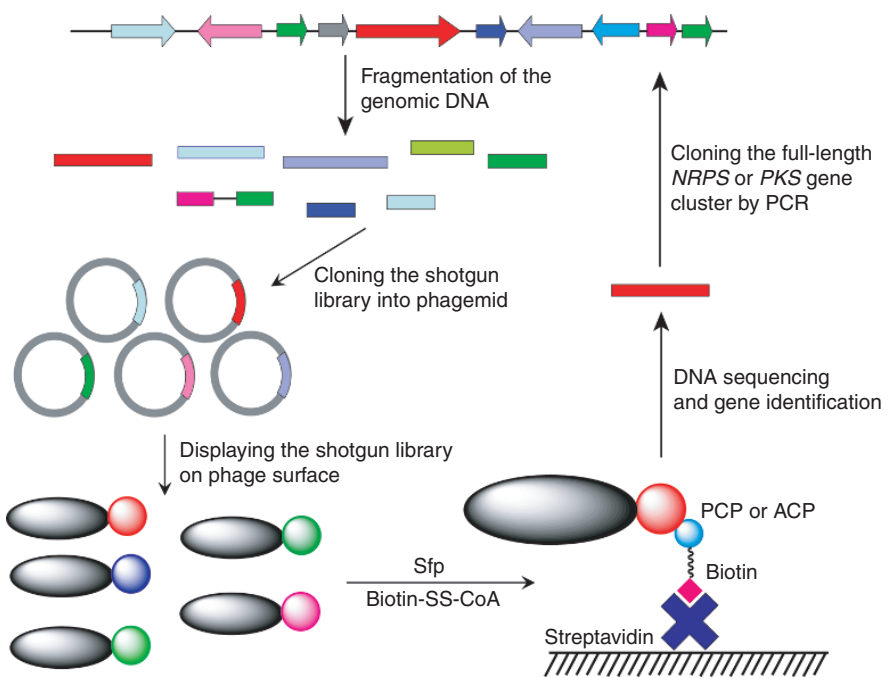

Figure 1 | High-throughput cloning of bacterial carrier proteins. Genomic libraries are displayed on phage, specifically biotinylated in vitro and isolated by iterative selection on streptavidin. Reprinted with permission from Elsevier.

researchers had previously shown that Sfp, a phosphopantetheinyl transferase from Bacillus subtilis, can efficiently biotinylate the carrier proteins from these pathways in vitro, using biotinylated coenzyme A as a sub- strate. "We were actually interested in finding a way to do protein labeling," says Yin, "but my background is in phage display, so my immediate tendency when something can be biotinylated is that this could be the basis

\section{PROTEOMICS}

\section{FROM SPECTRAL NETWORKS TO SHOTGUN SEQUENCING}

Researchers demonstrate a new paradigm for mass spectrometry-based peptide identification and de novo protein sequencing.

Most researchers in the mass spectrometry (MS)-based proteomics field take it for granted that at some point, they are going to need to do a database search to match the mass spectra of their peptides with those in a database to identify the peptide sequences and by extension, their parent proteins. But this process becomes difficult and painfully slow for peptides containing multiple post-translational modifications (PTMs). It also becomes pretty much impossible to identify proteins from organisms with unsequenced genomes, for which neither sequence nor spectral databases exist.

So what is a curious researcher to do? If you are Pavel Pevzner, a computer scientist at the University of California, San Diego, you think of something a bit out of the ordinary. Pevzner and his graduate student Nuno Bandeira recently reported a strategy to perform database searching without ever comparing a spectrum to a database (Bandeira et al., 2007a).

Rather than search a database to interpret a peptide mass spectrum, Bandeira, Pevzner and their coworkers developed the concept of spectral networks, using spectral alignment to discover related spectra. For example, two versions of the same peptide, one that contains post-translational modifications, and one without, will have related spectra, as will peptides (born of the same protein) with overlapping sequences. Pevzner explains the concept with an analogy: “Suppose you started from hundreds of spectra that are not related; they're kind of like cities. You are connecting them by roads. And all of a sudden, the spectra make sense because when they are connected by roads, you can use neighbors to interpret what is in every city."

To illustrate just how powerful the concept is, Bandeira and Pevzner combined efforts with Karl Clauser of the Broad Institute to demonstrate how spectral networks can be used to reconstruct protein sequences from unpurified mixtures of unknown proteins (Bandeira et al., 2007b). They apply a variety of proteases with different specificities to generate peptides with overlapping sequences. They use the spectral network of the overlapping peptide fragments to construct a 'virtual' MS/MS spectrum of very high quality, which can then be used to determine the sequence of the whole protein.

Bandeira and Pevzner investigated the venom of the western diamondback rattlesnake, as an example of a potentially medically important proteome from an organism for which the genome sequence has yet to be determined. Not only did they demonstrate for the first time that de novo protein sequencing 
for a powerful selection system."

This is exactly what the scientists went on to develop. They took a shotgun approach to cloning carrier proteins that are part of the polyketide and nonribosomal polypeptide synthetic pathways in $B$. subtilis, as well as in the myxobacterium Myxococcus xanthus. They displayed shotgun libraries of the bacterial genome on the surface of M13 phage, subjected the expressed proteins to in vitro biotinylation and fished out putative carrier proteins by iterative selection (Fig. 1). After sequencing the genes encoding these proteins, they mapped os them to the full-length clusters.

In B. subtilis, $85 \%$ of the genes recovered after five rounds of selection encoded carrier proteins of the relevant biosynthetic pathways; in all, $50 \%$ of the known carrier protein domains were cloned from a single genomic library. The M. xanthus genome has not been fully annotated, but here as well 22 carrier protein-encoding inserts were recovered, including as yet unannotated genes, in addition to six new sequences. As Sfp is known to have a fairly wide substrate specificity, and because it is only one of a family of enzymes that could be used for carrier protein selection, it is likely that this approach will be useful in several other bacterial species.

Undoubtedly, the application of this approach to genomes from unsequenced and ultimately from uncultured microorganisms will pose substantial new challenges. But Yin is optimistic. "I hope we can find a new cluster," he says. "The thing is, there is so little research on this. Even random sequencing of metagenomic samples has turned up some domains that may be involved in synthesizing new natural products, so I think we have a chance."

\section{Natalie de Souza}

\section{RESEARCH PAPERS}

Yin, J. et al. Genome-wide high-throughput mining of natural-product biosynthetic gene clusters by phage display. Chem. Biol. 14, 303-312 (2007).

from a crude biological mixture was possible, but importantly, "Because venom changes depending on the season of the year that it's collected, and geographical reasons [and so forth], we found single nucleotide polymorphism variants in the sample as well," says Bandeira.

Though slow and laborious, the present gold standard for protein sequencing is Edman degradation. "Implicitly, we have nothing against Edman degradation, but we feel that with this technique, Edman degradation becomes unnecessary," says Pevzner. "The number of amino acids we find in a single experiment is in the thousands;... with Edman degradation no one is able to reach anything close."

Bandeira and Pevzner are confident that their concept of spectral networks will become an important new paradigm in MSbased proteomics, as they have welcomed quite a bit of interest from new collaborators. "While we have demonstrated these methods for mixtures of proteins, these are still somewhat small mixtures of proteins," says Bandeira. "It will be exciting to see how these tools scale to whole proteomes."

\section{Allison Doerr}

\section{RESEARCH PAPERS}

Bandeira, N. et al. Protein identification by spectral networks analysis. Proc. Natl. Acad. Sci. USA 104, 6140-6145 (2007a).

Bandeira, N. et al. Shotgun protein sequencing: assembly of tandem mass spectra from mixtures of modified proteins. Mol. Cell. Proteomics; published online 19 April 2007b.

\section{NEWS IN BRIEF}

\section{CHEMICAL BIOLOGY}

\section{Genetically encodable aldehyde tag}

New bioorthogonal tags are always welcome additions to the chemical biologist's toolbox. Carrico et al. describe a new such handle, a genetically encodable aldehyde tag. A 6-amino-acid motif is recognized by formylglycine-generating enzyme, which oxidizes cysteine to the aldehyde-containing formylglycine. The aldehyde serves as a convenient attachment site for aminooxyand hydrazine-functionalized labels.

Carrico, I.S. et al. Nat. Chem. Biol.; published online 22 April 2007.

\section{PROTEOMICS}

\section{The Drosophila protein catalog}

Brunner et al. present a catalog of $63 \%$ of the predicted proteome of the model organism Drosophila melanogaster, using shotgun mass spectrometry. They obtained high coverage by using diverse samples, an extensive fractionation strategy and a statistical bioinformatic approach called analysis-driven experimentation, which allowed them to optimize experimental conditions to target under-represented portions of the proteome.

Brunner, E. et al. Nat. Biotechnol. 25, 576-583 (2007).

\section{GENE REGULATION}

\section{A tool to upregulate gene expression}

Whereas RNAi has come into its own as a means to knock down gene expression, no good corresponding tool has been available to do the opposite: upregulate gene expression. Xiao et al. describe the development of a cell-permeable synthetic transcription factor mimic, which activates gene expression in living cells by binding to a specially designed promoter. They were able to achieve a fivefold upregulation of expression in HeLa cells.

Xiao, X. et al. Angew. Chem. Int. Edn. 46, 2865-2868 (2007).

\section{SPECTROSCOPY}

\section{Aligning with nanotubes}

Liquid crystalline media are used to weakly align protein molecules during an NMR experiment, facilitating the measurement of residual dipolar couplings, which aid in structure solution. These media, however, are generally incompatible with the detergents used in membrane protein preparations. Douglas et al. report the design and synthesis of detergent-resistant DNA nanotubes as a viable alternative for the weak alignment of membrane proteins in NMR experiments.

Douglas, S.M. et al. Proc. Natl. Acad. Sci. USA, 104, 6644-6648 (2007).

\section{GENOMICS}

\section{A gene expression resource for fission yeast}

With the goal of understanding the multiple levels of regulation of gene expression, Lackner et al. present genome-wide data sets identifying key gene expression intermediates in the fission yeast Schizosaccharomyces pombe. Using microarray analysis, they collected data under standardized conditions, revealing new insights about systems-level regulation from transcription to translation.

Lackner, D.H. et al. Mol. Cell 26, 145-155 (2007). 